\title{
Polyarteritis nodosa: decreasing incidence in Poland
}

Krzysztof Kanecki ${ }^{1}$, Aneta Nitsch-Osuch ${ }^{1}$, Pawel Gorynski ${ }^{2}$, Waldemar Wierzba ${ }^{3}$, Patryk Tarka ${ }^{1}$, Piotr Tyszko ${ }^{4}$

${ }^{1}$ Department of Social Medicine and Public Health, Medical University of Warsaw, Warsaw, Poland

${ }^{2}$ National Institute of Public Health - National Institute of Hygiene, Warsaw, Poland ${ }^{3}$ University of Humanities and Economics, Lodz, Poland

${ }^{4}$ Institute of Rural Health, Lublin, Poland

Submitted: 7 October 2016

Accepted: 2 February 2017

Arch Med Sci 2019; 15 (5): 1308-1312

DOI: https://doi.org/10.5114/aoms.2017.68407

Copyright (C) 2017 Termedia \& Banach

\section{Abstract}

Introduction: There are many studies on the epidemiology of the orphan disease polyarteritis nodosa (PAN) in European countries. The use of a hospital morbidity database is an important element of epidemiological analysis. The study is the first such analysis in Poland and may provide an opportunity to compare the data in a European context.

Material and methods: We conducted a retrospective, population-based study using hospital discharge records with a PAN diagnosis. PAN incidence was estimated based on data from a Polish hospital morbidity study carried out by the National Institute of Public Health. Data on all inpatients discharged from non-psychiatric hospitals in Poland were collected between 2008 and 2013. We identified 557 patients with first-time hospitalizations for PAN.

Results: The sample consisted of 202 (36.3\%) males and 355 (63.7\%) females. The median and mean age was 53 and 50 years, respectively. The average annual incidence of PAN in Poland was estimated to be 2.4 per million. PAN incidence decreased significantly over time in Poland between 2008 and 2013 (3.3 per million vs 1.9 per million, $p<0.001$ ) and it may be associated with a decrease in hepatitis B incidence observed in Poland.

Conclusions: Incidence of PAN in Poland was estimated to be 2.4 per million and was comparable to the incidence found in other European countries. PAN incidence decreased during 2008-2013. The decrease in PAN incidence may be related to environmental, infectious (hepatitis B) or other unknown factors. Future research is needed to identify the cause of the decrease and potential triggers of the disease.

Key words: epidemiology, polyarteritis nodosa, rare disease.

\section{Introduction}

The term "orphan disease" is used for diseases diagnosed in fewer than 50 out of 100,000 patients. An example of an orphan disease in rheumatology is polyarteritis nodosa (PAN). Polyarteritis nodosa is a necrotizing arteritis of medium or small arteries; its symptoms do not include glomerulonephritis and inflammation of arterioles, capillaries and veins, and it is not related to the presence of anti-neutrophil cytoplasmic antibodies. The aetiology and pathogenesis of the disease are not known. A secondary form of the disease occurs in $>10 \%$ of cases and is associated with hepatitis B virus (HBV) or hepatitis C virus (HCV) infections.

\author{
Corresponding author: \\ Krzysztof Kanecki MD \\ Department of Social \\ Medicine and Public Health \\ 3 Oczki St \\ 02-007 Warsaw, Poland \\ Phone: +48 501929235 \\ E-mail: kanecki@mp.pl
}


According to the criteria for the diagnosis of PAN, the Chapel Hill Consensus Conference (CHCC 1994) produced definitions for the major types of vasculitis [1]. The second International Chapel Hill Consensus Conference changed names and definitions as appropriate and added important categories of vasculitis not included in CHCC 1994 [2]. The European League Against Rheumatism/ Paediatric Rheumatology International Trials Organisation/Paediatric Rheumatology European Society proposed validated classification criteria for childhood PAN [3, 4].

A number of studies have been carried out globally assessing PAN incidence. A 10-year study in the United Kingdom showed that the annual incidence of PAN, using the American College of Rheumatology criteria, was eight per million (95\% Cl: $5.5-11.2)$, and no cases of PAN as defined by CHCC 1994 were observed [5]. The annual incidence of PAN was 9.7 per million (95\% Cl: $7.0-$ 13.0) in Norwich (east England), which was higher than the incidence in Lugo (northwest Spain) of 6.2 per million (95\% Cl: 3.4-10.5) [6]. Within a defined population in southern Sweden, the annual PAN incidence per million population $(95 \% \mathrm{Cl})$ was estimated to be 0.9 (0-1.7) [7].

The point prevalence of PAN per million inhabitants was estimated on 1 January 2003 to be 31 (95\% Cl: 11-52) within a defined population in southern Sweden, and capture-recapture analysis estimated the completeness of the case findings to be $96 \%$ [8]. The prevalence per million adults was estimated to be 30.7 (95\% Cl: $21-40)$ for PAN in a French, multiethnic, urban population [9]. In the Australian Capital Territory and the surrounding rural region, annual PAN-specific incidences for each of two periods (i.e., 1995-1999 and 2000-2004) were 2.3 and 1.1 per million, respectively [10]. The estimated PAN incidence ranges from 2 to 9 per million individuals per year in adults in the United States [11]. Significantly higher PAN prevalence is observed in Europeans than in other ethnicities; this may be due to a genetic susceptibility common among Caucasians [9].

There is little information regarding the epidemiology of PAN in Poland. There is interest in rare autoimmune systemic vasculitis [12], or rare autoimmune multi-organ diseases that may be associated with hepatitis [13] in Poland. The major environmental factor associated with PAN is HBV infection [14]. It was reported that the prevalence of infection-associated PAN is related to the prevalence of the infections themselves [2].

This study was undertaken to assess the incidence of PAN in Poland and to analyse factors related to the epidemiology of this disease.

\section{Material and methods}

We conducted a retrospective, populationbased study using hospital discharge records with a PAN diagnosis. We analysed data on PAN patients hospitalized from 2008 to 2013 in Poland. Data were taken from an inpatient discharge database. The information was collected by the National Institute of Public Health as part of a Polish hospital morbidity study. Data on all inpatients discharged alive or dead from all hospitals, excluding psychiatric and military hospitals, were obligatorily sent to the institute, usually on a monthly basis. The data collected included information from hospital documentation about hospitalization with ICD10-coded diagnoses, date of admittance and discharge, birth date, sex, and code of place of residence. Collected data did not include patient names. Incidence rates were calculated using the number of PAN patients and corresponding census data. In addition to data from the hospital morbidity study, we obtained demographic data for the general Polish population from the Central Statistical Office in Poland [15].

Polyarteritis nodosa often requires hospitalization, and therefore analysis of hospitalizations may provide a good estimate of the incidence. In the United States, the incidence of another vasculitis, Kawasaki disease, has been best estimated from hospital discharge data [16].

\section{Statistical analysis}

Included in the study were all patients with a first-time hospitalization for PAN during the study period. All statistical analyses were performed using a licensed version of Statistica software (StatSoft, Inc., 2012) and WINPEPI (Abramson, J.H. WINPEPI updated: computer programs for epidemiologists, and their teaching potential. Epidemiologic Perspectives \& Innovations $2011,8: 1$ ). The results of descriptive analyses were expressed as means, medians and ranges for continuous variables and counts and percentages for categorical data. Assuming a Poisson distribution of the observed cases, $95 \%$ Cls were calculated. The $\chi^{2}$ test was used to determine whether there was a significant difference between the frequencies. The $\chi^{2}$ test was used for trend analysis. The $p$-value of $<0.05$ was considered to be statistically significant.

\section{Results}

Between 2008 and 2013, 1690 hospitalizations with a PAN diagnosis occurred. Of these, 557 represented a patient's first PAN diagnosis present in the medical records during the analysed period. The study sample comprised 202 (36.3\%) male patients and $355(63.7 \%)$ female patients. The 


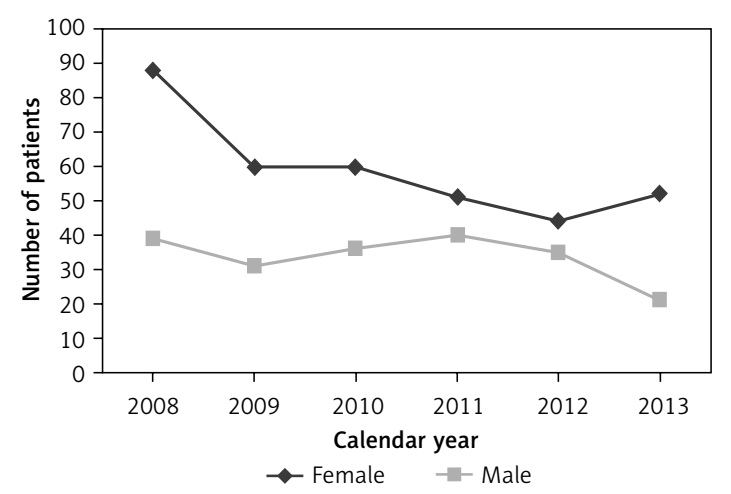

Figure 1. Incidence of polyarteritis nodosa in Poland by gender, 2008-2013

numbers of PAN cases per year are presented in Figure 1. Polyarteritis nodosa was significantly more common in females, $p<0.001$; the average $M$ : F ratio was 0.57 (95\% Cl: $0.41-0.77)$, and it ranged from 0.4 to 0.8 . The study sample comprised 494 (88.7\%) adult patients and 63 (11.3\%) patients younger than 18 years. The age distribution of female and male patients at their first hospitalization with a PAN diagnosis is presented in Figure 2. The median and mean age of all patients at first PAN hospitalization were 53 and 50 years, respectively. The incidence of PAN peaked in the 50-70 year age group. There were significant differences between people in more urban (384 patients) and more rural areas (173 patients; $p<0.001)$. The average annual incidence of PAN in Poland was estimated to be 2.4 per million (95\% Cl: 1.9-3.0). Polyarteritis nodosa incidence decreased significantly over time in Poland between 2008 and 2013 (3.3 per million vs. 1.9 per million, $p<0.001)$. The $\chi^{2}$ test for a trend confirmed the presence of a statistically significant trend of PAN incidence decrease in this study $(p<0.001)$. During the study period, we observed 15 deaths in PAN patients among all hospitalizations (10 females, 5 males; mean age 66 years, min.-max.: 2383 years) and deaths were observed in adults with no viral hepatitis diagnosis. In this group 9 deaths

A

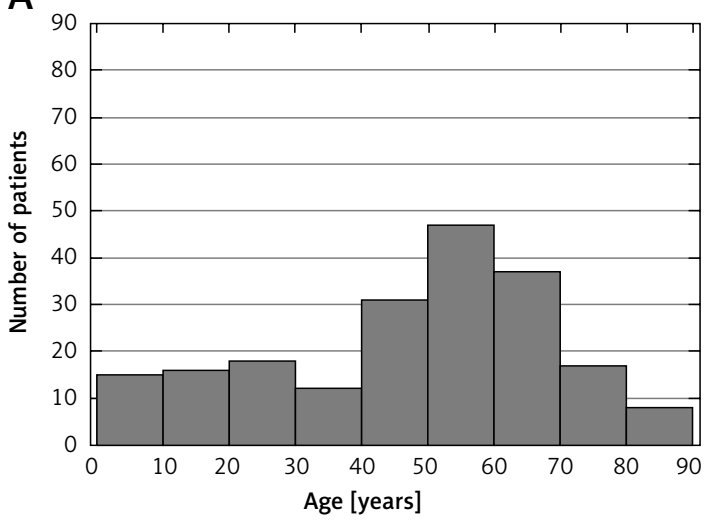

were observed in patients hospitalized with a firsttime diagnosis of PAN (7 females, 2 males; mean age 62 years, min.-max.: $23-83$ years). During the study period, we observed 4 cases of chronic viral hepatitis in PAN patients ( 1 viral hepatitis B, 2 viral hepatitis $C$, and 1 non- $B$, non- $C$ viral hepatitis cases). All cases of viral hepatitis were observed in adults ( 2 females, 2 males). Three out of 4 cases of viral hepatitis were observed in 2008, 1 in 2009, and none in 2010-2013.

\section{Discussion}

Epidemiological data on PAN incidence in European countries have been reported; however, to our knowledge, no large descriptive studies of the epidemiology of PAN in Poland existed prior to this analysis. Based on data from an inpatient discharge database, we estimate that the average annual incidence of PAN in Poland is 2.4 per million people. This finding is comparable with data from other European countries [5-7].

This study revealed a statistically significant decrease in PAN incidence in Poland in the analysed period. This may be related to unknown factors, such as changes in infection rates or demographics. The decreasing trend in PAN incidence may be related to vaccination strategies and awareness of blood-borne virus infections. It was suggested that the decline in PAN incidence paralleled better vaccination strategies and increased awareness of blood-borne virus screening in the United Kingdom [17]. Importantly, it was reported that the frequency of HBV-PAN has decreased in relation to improved blood safety and vaccination campaigns [18]. In another study it was also suggested that the decreasing incidence of HBV-associated PAN may be a consequence of vaccination campaigns and the improved safety of blood products [9].

In this study, we observed no patients with PAN and viral hepatitis during 2010-2013. In 2013, compared with the previous year, the incidence of HBV in Poland slightly decreased [19]. In contrast, the total number of hepatitis $C$ cases record-

B

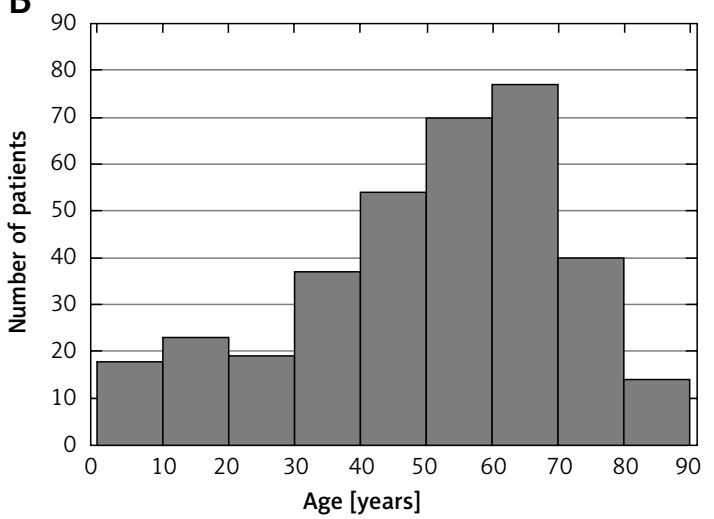

Figure 2. Age distribution of PAN patients in Poland by gender (A - male, B - female), 2008-2013 
ed during 2006-2013 in Poland was decreasing during 2006-2009 and slightly increasing during 2009-2013 [20].

Given that the association of hepatitis $B$ and hepatitis $C$ with PAN is well known, a review of the Polish epidemiological situation regarding these viruses is relevant. In Poland, there has been a spectacular improvement regarding hepatitis $B$ incidence rates due to the implementation of a very intensive and successful immunization program. The program began in 1984 and started with the introduction of hepatitis $B$ vaccination into the recommendations of the National Immunization Program; 'recommended' vaccine costs were paid by patients with no reimbursement. In 1989 , all newborns from HBV-infected mothers were offered a hepatitis $B$ vaccination free of charge, and in 1990 a three-dose vaccination schedule was introduced for health care workers and medical students. In 1994, universal, fully reimbursed vaccination for newborns and infants followed by catch-up vaccinations for adolescents was implemented [21]. As a result, the incidence rates for hepatitis B decreased from 40/100,000 in the early 1980 s to $4 / 100,000$ in 2013. In 2013, no cases of acute hepatitis $B$ were reported among people aged 0-24 years; the incidence rates were highest among people aged $65-74$ years $(0.44 / 100,000)$ and $40-44$ years $(13.3 / 100,000)$ [19]. It is a wellknown fact that PAN is more frequently reported in patients with chronic hepatitis B than in those with chronic hepatitis C [22]. We think that the significant decrease in hepatitis $B$ incidence in Poland as described above may be associated with the decreasing trend in PAN incidence in our country.

In this analysis, significantly more patients lived in urban areas than in rural ones. A higher frequency of PAN in urban populations was previously reported [9]. It was also reported that, among people from Poland with a chronic hepatitis B diagnosis, more were from urban populations than rural ones [19]. These observations may support the concept of infectious or environmental triggers for the disease. The idea that environmental factors may be important in the aetiopathogenesis of primary systemic vasculitis has been presented in the literature [23].

This study reveals that the epidemiology of PAN in Poland is unique, although it does exhibit similarities with other countries. Our finding of a 0.57 male-to-female ratio differs from findings from other countries. In other studies, males were reported to be more affected than females [24]. In one study, the male-to-female ratio was reported to be 0.88-1 [25]. In another study, there was no clear gender difference [8].

During the study period, we observed 15 deaths in PAN patients (9 in patients hospitalized with a first-time diagnosis of PAN). All deaths were observed in adults. We reviewed the published literature on the current risk of mortality in patients with PAN (survival rate of $75 \%$ to $80 \%$ at 5 years) [26]. The major cause of early death was uncontrolled vasculitis (58\%), followed by infection (26\%) [27]. The outcome of PAN is improved in patients receiving treatment; the 5 -year survival rate is approximately $80 \%$ [24]. Five-year relapse-free survival rates were $59.4 \%(95 \% \mathrm{Cl}: 52.6-67.0)$ vs. $67.0 \%(95 \% \mathrm{Cl}: 58.5-76.8)$ for non-HBV-related PAN and HBV-related PAN, respectively [24]. A multivariate analysis revealed that age $>65$ years, hypertension, and gastrointestinal manifestations requiring surgery or at least consultation with a surgeon were independent predictors of death, whereas patients with cutaneous manifestations or non-HBV-related PAN had a higher risk of relapse [24].

No deaths were observed in patients younger than 18 years. It is worth noting that, in a retrospective study of Caucasian children diagnosed with PAN, the worst outcomes were significantly correlated with renal and neurological involvement [28].

This study has several limitations. The database we used provided general information regarding hospitalizations of PAN patients, thereby allowing us to present an epidemiological report; however, it did not allow us to examine risk factors and other correlates of the disease in individual patients. An important limitation of this study is that it relied on discharge records from inpatient hospitalizations; it therefore excludes patients who were seen only as outpatients. Thus, our reported incidence rate may be an underestimate. The true rate could be much higher, as this type of vasculitis is a systemic necrotizing disease with multi-organ involvement and may require advanced diagnostic procedures that can be conducted in inpatient settings. Cutaneous PAN is a benign vasculitis, but it may require differentiation from systemic PAN in inpatient settings due to the different clinical course and management of the two conditions.

Additionally, the first appearance of a PAN diagnosis in the inpatient discharge database is not necessarily the date of first diagnosis. This inaccuracy may result in an overestimate of the count of incident cases. However, the long duration of observation in this study may minimize the overestimation.

In conclusion, incidence of PAN in Poland was estimated to be 2.4 per million patients and was comparable to the incidence found in other European countries. PAN incidence decreased during 2008-2013. The decrease in PAN incidence may be related to infectious, environmental or other unknown factors. Future research is needed to 
identify the cause of the decrease and potential triggers of the disease.

\section{Conflict of interest}

The authors declare no conflict of interest.

\section{References}

1. Jennette JC, Falk RJ, Andrassy K, et al. Nomenclature of systemic vasculitides. Proposal of an international consensus conference. Arthritis Rheum 1994; 37: 187-92.

2. Jennette JC, Falk RJ, Bacon PA, et al. 2012 revised International Chapel Hill Consensus Conference Nomenclature of Vasculitides. Arthritis Rheum 2013; 65: 1-11.

3. Ozen S, Pistorio A, Iusan SM, et al. EULAR/PRINTO/PRES criteria for Henoch-Schönlein purpura, childhood polyarteritis nodosa, childhood Wegener granulomatosis and childhood Takayasu arteritis: Ankara 2008. Part II: Final classification criteria. Ann Rheum Dis 2010; 69: 798-806.

4. Ruperto N, Ozen S, Pistorio A, et al. EULAR/PRINTO/PRES criteria for Henoch-Schönlein purpura, childhood polyarteritis nodosa, childhood Wegener granulomatosis and childhood Takayasu arteritis: Ankara 2008. Part I: Overall methodology and clinical characterisation. Ann Rheum Dis 2010; 69: 790-7.

5. Watts RA, Lane SE, Bentham G, Scott DG. Epidemiology of systemic vasculitis: a ten-year study in the United Kingdom. Arthritis Rheum 2000; 43: 414-9.

6. Watts R, Gonzalez-Gay M, Lane S, Garcia-Porrua C, Bentham G, Scott D. Geoepidemiology of systemic vasculitis: comparison of the incidence in two regions of Europe. Ann Rheum Dis 2001; 60: 170-2.

7. Mohammad AJ, Jacobsson LT, Westman KW, Sturfelt G, Segelmark M. Incidence and survival rates in Wegener's granulomatosis, microscopic polyangiitis, Churg-Strauss syndrome and polyarteritis nodosa. Rheumatology (Oxford) 2009; 48: 1560-5.

8. Mohammad AJ, Jacobsson LT, Mahr AD, Sturfelt G, Segelmark M. Prevalence of Wegener's granulomatosis, microscopic polyangiitis, polyarteritis nodosa and Churg-Strauss syndrome within a defined population in southern Sweden. Rheumatology (Oxford) 2007; 46: 1329-37.

9. Mahr A, Guillevin L, Poissonnet M, Ayme S. Prevalences of polyarteritis nodosa, microscopic polyangiitis, Wegener's granulomatosis, and Churg-Strauss syndrome in a French urban multiethnic population in 2000: a capture-recapture estimate. Arthritis Rheum 2004; 51: 92-9.

10. Ormerod AS, Cook MC. Epidemiology of primary systemic vasculitis in the Australian Capital Territory and south-eastern New South Wales. Intern Med J 2008; 38: 816-23.

11. Watts RA, Scott DGI. Epidemiology of vasculitis. In: Vasculitis. $2^{\text {nd }}$ ed. Ball GV, Bridges SL (eds.). Oxford University Press, Oxford 2008; 7-21.

12. Woźniacka A, Sysa-Jędrzejowska A, Jurowski P, Jabłkowski M, Kot M. Morbus Behçet - a rare disease in Central Europe. Arch Med Sci 2015; 11: 1189-96.

13. Białkowska J, Zygmunt A, Lewiński A, et al. Hepatitis and the polyglandular autoimmune syndrome, type 1 . Arch Med Sci 2011; 7: 536-9.

14. De Virgilio A, Greco A, Magliulo G, et al. Polyarteritis nodosa: a contemporary overview. Autoimmun Rev 2016; 15: $564-70$
15. Central Statistical Office of Poland. Available from: http://stat.gov.pl [Accessed: 2014-10-13].

16. Newburger JW, Takahashi M, Gerber MA, et al. Diagnosis, treatment, and long-term management of Kawasaki disease: a statement for health professionals from the Committee on Rheumatic Fever, Endocarditis and Kawasaki Disease, Council on Cardiovascular Disease in the Young, American Heart Association. Pediatrics 2004; 114: 1708-33.

17. Barlow EL, Seddon O, Healy B. PAN's labyrinth: a multidisciplinary delayed diagnosis and patient's perspective. BMJ Case 2016, doi: 10.1136/bcr-2015-213495.

18. Guillevin L, Mahr A, Callard P, et al. Hepatitis B virus-associated polyarteritis nodosa: clinical characteristics, outcome, and impact of treatment in 115 patients. Medicine (Baltimore) 2005; 84: 313-22.

19. Piwowarow K, Stepien M. Hepatitis B in Poland in 2013. Przegl Epidemiol 2015; 69: 251-6.

20. Stepien M, Rosinska M. Hepatitis C oubreaks in Poland in 2003-2013. Medical procedures as a dominant route of HCV transmission. Przegl Epidemiol 2015; 69: 465-72.

21. Magdzik W. Proposals concerning ways of control of hepatitis B in Poland since 2008 [Polish]. Przegl Epidemiol 2007; 61: 113-21.

22. Nishida N, Kudo M. Clinical features of vascular disorders associated with chronic hepatitis virus infection. Dig Dis 2014; 32: 786-90.

23. Watts R, Lane $S$, Scott $D$, et al. Epidemiology of vasculitis in Europe. Ann Rheum Dis 2001; 60: 1156-7.

24. Pagnoux C, Seror R, Henegar C, et al. Clinical features and outcomes in 348 patients with polyarteritis nodosa: a systematic retrospective study of patients diagnosed between 1963 and 2005 and entered into the French Vasculitis Study Group Database. Arthritis Rheum 2010; 62: 616-26.

25. Jokar M, Mirfeizi Z. Epidemiology of vasculitides in Khorasan Province, Iran. Iran J Med Sci 2015; 40: 362-6.

26. Phillip R, Luqmani R. Mortality in systemic vasculitis: a systematic review. Clin Exp Rheumatol 2008; 26 Suppl 51: 94-104.

27. Bourgarit A, Le Toumelin P, Pagnoux C, et al. Deaths occurring during the first year after treatment onset for polyarteritis nodosa, microscopic polyangiitis, and Churg-Strauss syndrome: a retrospective analysis of causes and factors predictive of mortality based on 595 patients. Medicine (Baltimore) 2005; 84: 323-30.

28. Falcini F, La Torre F, Vittadello F, et al. Clinical overview and outcome in a cohort of children with polyarteritis nodosa. Clin Exp Rheumatol 2014; Suppl 82: 134-7. 